\title{
A Serine/Threonine Kinase PknL is required For the Persistence of Mycobacterium tuberculosis
}

\author{
Ahmed Kabir Refaya* and Sujatha Narayanan \\ Department of Immunology, National Institute for Research in Tuberculosis, Mayor Sathiyamoorthy road, Chetpet, \\ Chennai, India
}

Received: 21 December, 2017; Accepted: 02 March, 2018; Published: 13 April, 2018

*Corresponding author: Ahmed Kabir Refaya, Department of Immunology, National Institute for Research in Tuberculosis, Mayor Sathiyamoorthy road, Chetpet, Chennai-600031, India, Tel: +91-44-2836 9628, 9500; E-mail: refayanasar@gmail.com

\begin{abstract}
Latent tuberculosis infection is mainly characterized by the existence of $M$. tuberculosis within the nutrient starved environment of caseous lung granulomas. Little is known about the survival niche of $M$. tuberculosis and its non-replicating nature which mimics the state of latent infection. The presence of 11 serine/threonine protein kinases and a similar number of two-component systems signifies the importance of the two signal transduction mechanisms in the adaptation of this bacterial pathogen to its environment. PknL, a serine/threonine kinase is well known for its role in the adaptation of $M$. tuberculosis. In this study we have investigated the role of $P k n L$ in starvation response by comparing the growth of previously constructed knockdown (PknL-AS) and over expressed (PknL-S) strains of $P k n L$ along with the Wild type strain $\left(\mathrm{H}_{37} \mathrm{Rv}\right)$ in different nutrient starved conditions and also monitored the revival ability of these strains upon transferring them again into enriched media. We have also assessed the intracellular growth and cytokine profile of these strains in THP-1 cell line. Irrespective of the medium of starvation, we observed a significant decrease in the growth and viability of PknL-AS. The intracellular growth of PknL-AS in THP-1 cell significantly decreased compared to the other strains. Taken together our data conclusively point to the fact that $P k n L$ is required for the persistence of Mycobacterium tuberculosis in the host.
\end{abstract}

Keywords: Nutrient acquisition; PknL; Persistence; Starvation models; survival;

\section{Importance}

Latency is associated with a state where the bacilli can neither die nor replicate but can only survive inside the granulomas until it encounters a favorable condition. This study has employed different type of starvation models to show that a serine/ threonine kinase $P k n L$ is responsible for both nutrient acquisition and also for the reactivation of Mycobacterium tuberculosis after starvation. Further we also assessed the intracellular survival of the pathogen in THP-1 macrophages and found that PknL is essential for the growth of the pathogen. Hence we claim that $P k n L$ is required for the persistence of Mycobacterium tuberculosis inside the host.

\section{Introduction}

Almost one-third of the world's population is affected with latent $M$. tuberculosis infection providing a large reservoir for the disease to reactivate and $2-10 \%$ of latently infected individuals are estimated to reactivate the disease with an increase of $>20$ folds in case of HIV co-infection [1]. It is a scenario where the host immune system arrests the infection of mycobacteria transmitted via respiratory route by forming a granuloma. It is in this granuloma, M. tuberculosis is encountered with conditions like hypoxia, nutrient starvation, $\mathrm{pH}$ change and free radicals which in turn induce the mycobacteria to shift to a non-replicative state. This state of dormancy in a bacilli are usually characterized by its slow in vitro growth, a downshift of metabolic pathways, altered staining features, an inability to be cultivated on solid media, and resistance to anti-mycobacterial agents. In vitro models using conditions of reduced oxygen, nutrient deprivation and stationary phase survival have demonstrated that $M$. tuberculosis is able to survive for extended periods in a non-replicating state.

Wayne's model of oxygen deprivation mimics the 'dormant' state in vitro [2]. This model is vastly used to identify genes and protein induced in M. tuberculosis and M. bovis BCG during oxygen limitation $[3,4]$. Many evidences pertain to the fact that persistent bacilli in lung lesions suffer nutrient deprivation. Altered colony morphology and staining properties were observed in M. tuberculosis isolated from the lung lesions compared with bacilli grown in vitro. The growth and acid fast staining property of $M$. tuberculosis were restored when they were transferred to nutrient-rich medium in-spite of being starved for almost 2 years in distilled water [5]. Another type of nutrient starvation model was designed by Loeble, which involves transfer of cultures from nutrient-rich medium into Phosphate-Buffered Saline (PBS) and the respiration rates were measured using a manometer. A gradual shutdown of respiration and growth to minimal levels were observed, but the bacilli remained viable and were able to recover when returned to rich medium [6]. Modified 7H9 media lacking phosphate, carbon and nitrogen have also been extensively used to study the stringent response of the bacilli and also to characterize genes influenced by these nutrients [7-9].

The virulence of M. tuberculosis is generally assessed by their intracellular survival within macrophages or using suitable animal model. Various studies in the past have demonstrated 
the rapid growth of virulent strains compared to it's a virulent counterpart [10]. The recognition of $M$. tuberculosis by macrophages leads to cellular activation and rapid production of pro-and anti-inflammatory cytokines. The successful outcome of mycobacterial infections depends upon the cytokine networks established and maintained by macrophages.

We have previously generated mutant strains of $P k n L$ by antisense strategy in an IPTG inducible system and have also reported that this gene is involved in the adaptive response of Mycobacterium tuberculosis [11]. In this study, we have used the same constructs to determine its role in starvation survival response of the pathogen along with the wild type $\mathrm{H}_{37} \mathrm{Rv}$. For this we developed different models of starvation which include Complete Nutrient Starvation (CNS), Carbon Starvation (CS), Phosphate Starvation (PS) and Nitrogen Starvation (NS) for evaluating the growth and survival of PknL-S, PknL-AS along with Wild type $\left(\mathrm{H}_{37} \mathrm{Rv}\right)$ strains. The viability of all the strains were assessed by transferring these nutrient deprived and nonreplicating strains to nutrient rich medium at various time points. Intracellular viability of these strains were also determined by infecting them in macrophages and determining the survival rate. We have also explored the expression pattern of cytokines such as IL- 6 and IL-12p40 among these strains.

\section{Materials and Methods}

\section{Bacterial strains and Culture conditions}

All the strains Wild type $\mathrm{H}_{37} \mathrm{Rv}$, PknL-S and PknL-AS were grown in 7H9 (Middlebrooks) medium supplemented with $10 \%$ ADS (Albumin Dextrose Saline) and 0.05\% Tween80. Hygromycin $(50 \mathrm{mg} / \mathrm{ml})$ and IPTG $(10 \mathrm{mM})$ was added when required. 1XPBS was used for Complete Nutrient Starvation (CNS). Middlebrooks 7H9 supplemented with dextrose free ADS and $0.025 \%$ of Tylaxopol lacking glycerol was used as Carbon Starvation (CS) model [12]. For Phosphate Starvation (PS), modified 7H9 medium (Sodium Citrate, pyridoxine, biotin, ferric ammonium citrate, magnesium sulfate, calcium chloride, zinc sulfate and copper sulfate) lacking phosphate buffering components was prepared and the $\mathrm{pH}$ was adjusted to 6.6 with MOPS [7]. In case of Nitrogen Starvation (NS) modified 7H9 medium was supplemented with either glutamic acid (NSG) or ammonium sulfate (NSA) as the nitrogen source. In addition to these starvation models, Middle brooks 7H9 media supplemented with Methionine Sulfoximine (MetSox), a potent glutamine inhibitor was also used as one of the nitrogen starvation model (NSMetSox).

In-vitro growth and survival determinations in completely starved (CNS) medium

All the strains Wild type, PknL-S and PknL-AS were initially grown in Middlebrooks $7 \mathrm{H} 9$ broth supplemented with $0.5 \%$ glycerol, $0.05 \%$ Tween 80 , and $10 \%$ albumin, dextrose along with $50 \mu \mathrm{g} / \mathrm{ml}$ of Hygromycin and 10mM IPTG for induction. The log phase cultures were washed twice with 1XPBS (CNS) and diluted to an $\mathrm{OD}_{600}$ of 0.2 with the same along with $50 \mu \mathrm{g} / \mathrm{ml}$ of hygromycin and $10 \mathrm{mM}$ IPTG. Diluted cultures were then grown in the shaker incubator at $200 \mathrm{rpm}$ and at $37^{\circ} \mathrm{C}$. Aliquots of the cultures were withdrawn at regular intervals on day $0,2,5,7$, 10,14 and 21 and the growth was monitored by measuring the culture OD at $600 \mathrm{~nm}$ using SpectraMax 250 microplate reader (Molecular Devices). Simultaneously, the survival or viability of all the strains were analyzed by plating serially diluted cultures from the starved medium on the 7H10-ADS enriched plates at every time point. CFU measurements were made after incubation of the plates at $37^{\circ} \mathrm{C}$ for $4-5$ weeks.

\section{In-vitro growth and survival determinations in Phosphate (PS) \& Carbon (CS) starved medium}

All the strains Wild type, PknL-S and PknL-AS were initially grown in Middle brooks $7 \mathrm{H} 9$ broth supplemented with $0.5 \%$ glycerol, $0.05 \%$ Tween 80 , and $10 \%$ albumin, dextrose along with $50 \mu \mathrm{g} / \mathrm{ml}$ of Hygromycin and 10mM IPTG for induction. The log phase cultures were washed twice with either Phosphate Starved (PS) or carbon deprived (CS) medium and diluted to an $\mathrm{OD}_{600}$ of 0.2 with the respective medium along with $50 \mu \mathrm{g} / \mathrm{ml}$ of hygromycin and 10mM IPTG. Diluted cultures were then grown in the shaker incubator at $200 \mathrm{rpm}$ and at $37^{\circ} \mathrm{C}$. Aliquots of the cultures were withdrawn at regular intervals on day $0,2,5,7$, 10,14 and 21 and the growth was monitored by measuring the culture OD at $600 \mathrm{~nm}$ using SpectraMax 250 microplate reader (Molecular Devices). Simultaneously, the survival or viability of all the strains were analyzed by plating serially diluted cultures from the respective starved medium on the 7H10-ADS enriched plates at every time point. $\mathrm{CFU}$ measurements were made after incubation of the plates at $37^{\circ} \mathrm{C}$ for $4-5$ weeks.

\section{In-vitro growth and survival determinations in different sources of Nitrogen starved medium}

All the strains Wild type, PknL-S and PknL-AS were initially grown in Middlebrooks 7H9 broth supplemented with $0.5 \%$ glycerol, $0.05 \%$ Tween 80 , and $10 \%$ albumin, dextrose along with $50 \mu \mathrm{g} / \mathrm{ml}$ of Hygromycin and 10mM IPTG for induction. The log phase cultures were washed twice with respective nitrogen deficient medium NSG and NSA and diluted to an $\mathrm{OD}_{600}$ of 0.2 with same medium along with $50 \mu \mathrm{g} / \mathrm{ml}$ of hygromycin and $10 \mathrm{mM}$ IPTG. Diluted cultures were then grown in the shaker incubator at $200 \mathrm{rpm}$ and at $37^{\circ} \mathrm{C}$. Aliquots of the cultures were withdrawn at regular intervals on day $0,2,5,7,10,14$ and 21 and the growth was monitored by measuring the culture OD at $600 \mathrm{~nm}$ using SpectraMax 250 microplate reader (Molecular Devices). Simultaneously, the survival or viability of all the strains were analyzed by plating serially diluted cultures from the starved medium on the 7H10-ADS enriched plates at every time point. $\mathrm{CFU}$ measurements were made after incubation of the plates at $37^{\circ} \mathrm{C}$ for $4-5$ weeks.

\section{In-vitro growth and survival determinations in Nitrogen starved medium by inhibiting Glutamine Synthetase (GS)}

All the strains Wild type, PknL-S and PknL-AS were grown in enriched Middle brooks 7H9 broth supplemented with $0.5 \%$ glycerol, $0.05 \%$ Tween 80 , and $10 \%$ albumin, dextrose along with $50 \mu \mathrm{g} / \mathrm{ml}$ of Hygromycin until log phase $\left(\mathrm{OD}_{600} 0.4-0.5\right) .10 \mathrm{mM}$ 
IPTG was used as inducer. At this stage $200 \mu \mathrm{M}$ of L-Methionine Sulfoximine (MetSox) was added to these cultures [13]. This was considered as day 0 time point. Aliquots of the cultures were withdrawn and the growth was measured and simultaneously the serially diluted cultures were placed on 7H10-ADS plates and in 7H10-ADS-MetSox plates. The cultures were then grown in the shaker incubator at $200 \mathrm{rpm}$ and at $37^{\circ} \mathrm{C}$. Aliquots of the cultures were withdrawn at regular intervals on consecutive days $2,5,7$, 10,14 and 21 and the growth was monitored by measuring the culture OD at $600 \mathrm{~nm}$ using Spectra Max 250 microplate reader (Molecular Devices). The viability of all the strains were checked by plating serially diluted cultures from the starved medium on the 7H10-ADS and 7H10-ADS-MetSox plates at every time point. CFU measurements were enumerated after incubation of the plates at $37^{\circ} \mathrm{C}$ for $4-5$ weeks.

\section{Intracellular viability of PknL-AS, PknL-S and Wildtype strains in THP-1 macrophage}

Intracellular viability of the Wild type and the mutant strains were determined using macrophage infection studies. THP-1 cells (National center for cell sciences, Pune, India) were grown in RPMI -1640 (Gibco, Invitrogen) supplemented with 10\% fetal bovine serum (FBS) and antimicrobials and anti-mycotic agents (Gibco, Invitrogen). THP-1 cells were differentiated with $50 \mathrm{nM}$ phorbol 12-myristate13-acetate for 72 hours and allowed to recover for 24 hours prior to infection. Macrophages were then infected in triplicates with PknL-AS, PknL-S and Wild type strain at an MOI of 1:10 and three wells of uninfected control was also included in each time point of the experiment. IPTG was added in $0 \mathrm{mM}, 1 \mathrm{mM}$ and $10 \mathrm{mM}$ for induction. Phagocytosis was allowed to take place for $4 \mathrm{hrs}$. After which mono layers were washed with RPMI to remove non-phagocytosed or non-adherent bacilli. The infected macrophages were then incubated with fresh RPMI1640 supplemented with $10 \% \mathrm{FBS}$ at $37^{\circ} \mathrm{C}$ in the presence of $5 \% \mathrm{CO}_{2}$. Intracellular bacilli were recovered by lysing infected macrophages and monocytes with $1 \%$ ice cold trypsin on day 0 (after 4 hrs of infection) and 1, 3, $5 \& 7$ days post infection. Bacterial load was then estimated by plating serial dilutions of the lysates onto Middle brooks 7H10 plates with 10\% OADC and supplemented with $0 \mathrm{mM}, 1 \mathrm{mM}$ and $10 \mathrm{mM}$ concentration of IPTG. The viable counts were determined after 4-6 weeks of incubation at $37^{\circ} \mathrm{C}$. Simultaneously, the cell free culture supernatants were collected and stored at $-80^{\circ} \mathrm{C}$ for cytokine analysis.

\section{Cytokine analysis by ELISA}

Cell-free culture supernatants collected from the infected cell lines (THP-1 macrophage) with the Wild type and mutant strains at different time points were analyzed for the levels of IL- 6 and IL-12p 40 cytokines by sandwich ELISA using BP optEIA ELISA kit (BD pharmingen, USA) as per manufacturer's protocol.

\section{Statistical Analysis}

Two-way ANOVA with repeated measures using Bonferroni post-test was done for all the experiments including growth and survival kinetics, intracellular growth and cytokine analysis. GraphPad Prism 5.0 Software was used for all the tests and $p$-value $<0.05$ were considered significant.

\section{Results \\ In vitro growth kinetics and survival pattern in completely starved medium}

To determine the effect of antisense inhibition of $P k n L$ on rate of growth and viability of the strains in the absence of all nutrients, the growth profile of Wild type, PknL-S and PknL-AS were compared by monitoring the $\mathrm{OD}_{600}$ in starved media (CNS) and the viability of the strains were assessed by comparing the Colony Forming Units (CFU) in nutrient rich 7H10 agar supplemented with OADC at $0,2,5,7,10,14$ and 21 days. There was a clear lysis observed in all the three strains in CNS media (Figure 1 a). After day 10 there was a slight increase in growth in case

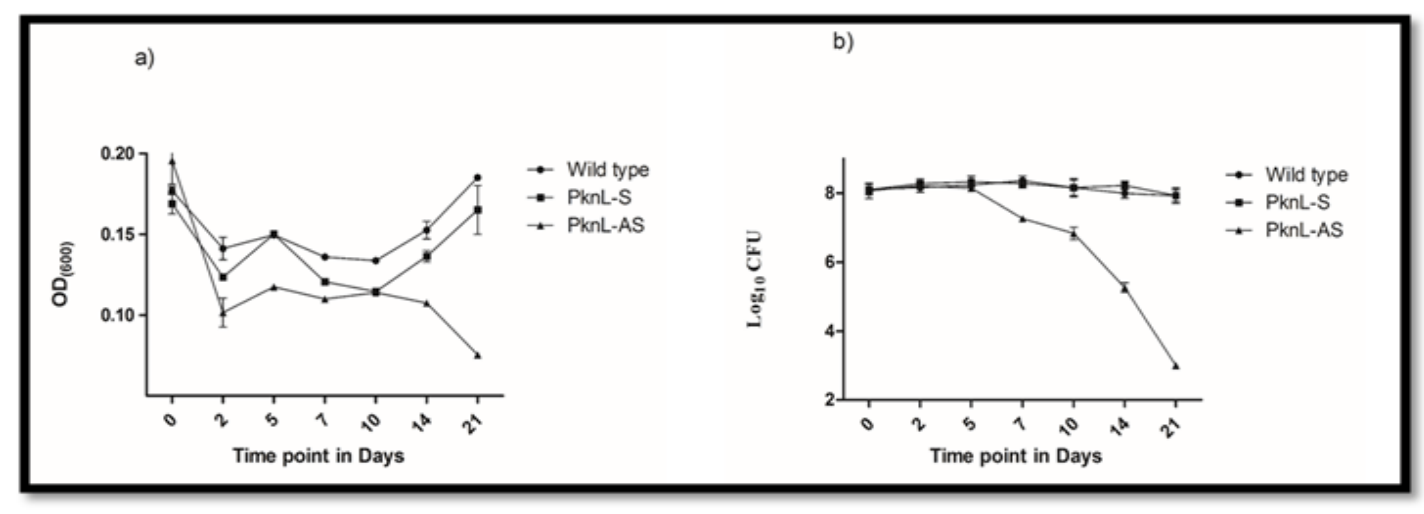

Figure 1: In vitro growth and survival kinetics in completely starved medium (CNS)

Growth and viability of the strains Wild type, PknL-S and PknL-AS in CNS media were monitored by measuring the $\mathrm{OD}_{600}$ and CFU obtained from serial dilutions from enriched 7H10 Agar plates with $10 \mathrm{mM}$ IPTG on 0,2,5,7,10,14 \& 21 days. The data is representative of three independent experiments. Error bars represent the standard error of the means. The graph represents the

a) $\mathrm{OD}_{600}$ plotted against the respective time points in CNS media and

b) $\log 10(\mathrm{CFU} / \mathrm{ml})$ of the strains grown in enriched $7 \mathrm{H} 10$ agar plates.

There was a significant decrease $(p<0.05$ to $p<0.001)$ in the viability of PknL-AS from day 10 onwards compared to PknL-S and wild type. 
of Wild type and PknL-S whereas PknL-AS was unable to grow in the absence of nutrients. The viability of the strains exhibit the in vivo phenomenon in reactivation of the Mycobacteria when it encounters a favorable environment (nutrient rich). The reactivating capacity of the strains also exhibited a similar pattern wherein all the strains were able to revive its growth in nutrient rich medium except PknL-AS which was unable to revive its growth after day 10 and the difference was significant $(p<0.05$ to $p<0.001$ ). There was no colony growth observed on agar plates for PknL-AS on day 21, hence for calculation purpose the colony growth was considered as 1 (Figure $1 b$ ).

\section{In vitro growth kinetics and survival pattern in phosphate} depleted medium

To determine the effect of antisense inhibition of PknL on rate of growth and viability of the strains in the absence of phosphate, the growth profile of Wild type, PknL-S and PknL- AS was compared by monitoring the $\mathrm{OD}_{600}$ in PS and the viability of the strains were assessed by comparing the CFU in nutrient rich 7H10 agar supplemented with OADC at 0, 2,5,7,10,14 and 21 days. In the absence of phosphate all the strains showed a sustained growth until day 5 followed by a steep decline on day 7 and again an increase in growth was noticed for all the three strains. Compared with Wild type and PknL-AS, PknL-S showed a significant increase $(p<0.05)$ in growth after day 10 (Figure $2 a)$. The reactivation ability of all the strains in the presence of nutrient rich 7H10 Agar were viable until day 5, but PknL-AS showed a significant decrease $(p<0.01$ to $p<0.001)$ compared to PknL-S and Wild type on day $10 \& 21$ (Figure 2 b).

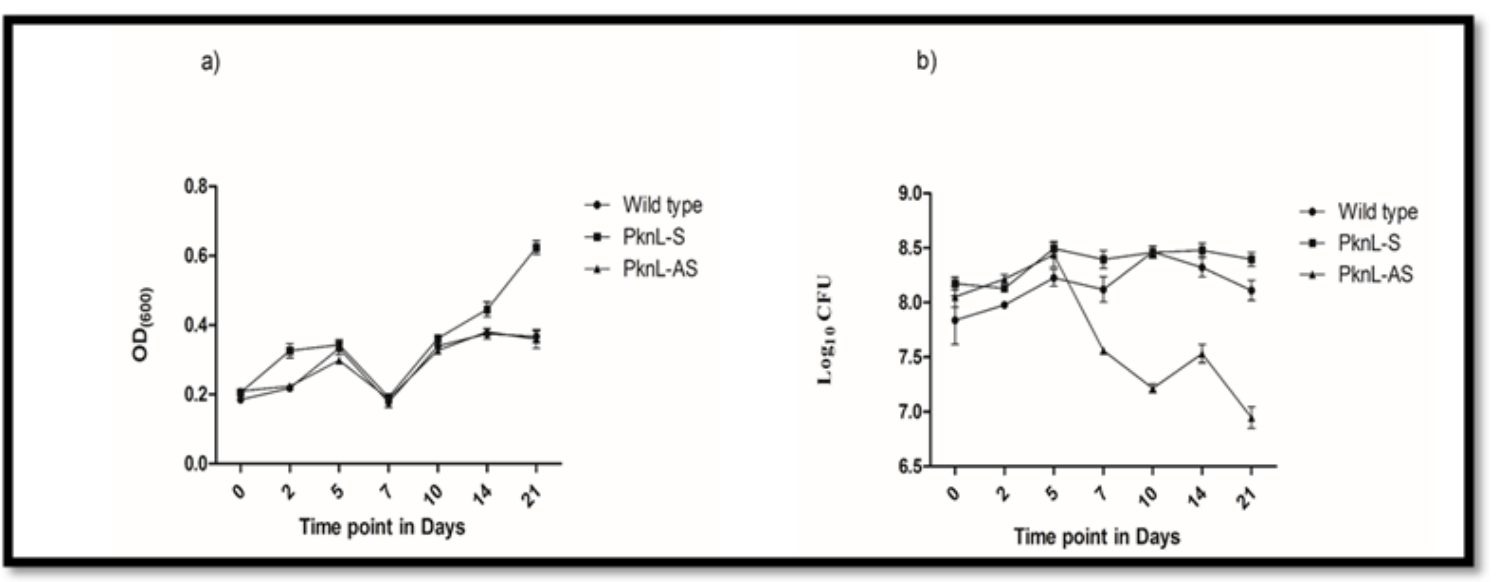

Figure 2: In vitro growth and survival kinetics in Phosphate starved medium (PS)

Growth and viability of the strains Wild type, PknL-S and PknL-AS in PS media were monitored by measuring the $\mathrm{OD}_{600}$ and CFU obtained from serial dilutions from enriched 7H10 Agar plates with 10mM IPTG on 0,2,5,7,10,14 \& 21 days. The data is representative of three independent experiments. Error bars represent the standard error of the means. The graph represents the

a) $O D_{600}$ plotted against the respective time points in PS media and

b) $\log 10(\mathrm{CFU} / \mathrm{ml})$ of the strains grown in enriched $7 \mathrm{H} 10$ agar plates.

There was a significant increase $(p<0.05)$ in the growth of PknL-S compared to Wild type and PknL-AS day 21. The viability of PknL-AS in nutrient rich 7H10 solid medium showed a significant decrease $(p<0.01$ to $p<0.001)$ compared to PknL-S and Wild type on day $10 \& 21$.

\section{In vitro growth kinetics and survival pattern in carbon starved medium}

In order to assess the growth and viability of the strains in the absence of carbon, the growth profile of Wild type, PknL-S and PknL- AS was compared by monitoring the $\mathrm{OD}_{600}$ in carbon starved media (CS) and the viability of the strains were assessed by comparing the CFU in nutrient rich 7H10 agar supplemented with OADC at 0,2,5,7,10,14 and 21 days. All the strains were able to grow in the absence of carbon, but growth of PknL-AS was a little retarded when compared to Wild type and PknL-S (Figure 3a). Although, the retarded growth of PknL-AS was not significant, it did have an effect on the viability of the strain, as PknL-AS was not able to revive back its growth rate as Wild type and PknL-S and significantly $(P<0.05)$ lagged behind in reactivation (Figure $3 b)$.

\section{In vitro growth kinetics and survival pattern in Nitrogen starved medium (NSA)}

In order to assess the growth and viability of the strains in the presence of Ammonia as the sole nitrogen source, the growth profile of Wild type, PknL-S and PknL- AS was compared by monitoring the $\mathrm{OD}_{600}$ in modified 7H9 medium, containing only an inorganic source of nitrogen i.e. Ammonium Sulfate (NSA) and the viability of the strains were assessed by comparing the CFU obtained after transferring the serially diluted cultures of starved medium in nutrient rich 7H10 agar supplemented with OADC at $0,2,5,7,10,14$ and 21 days. All the strains showed a similar growth profile in the presence of Ammonium sulfate, but PknLAS showed a slightly enhanced (not significant) growth after day 10 compared to Wild type and PknL-S (Figure 4a). Similarly, the viability of the strains was also very similar, as all the strains Wild 


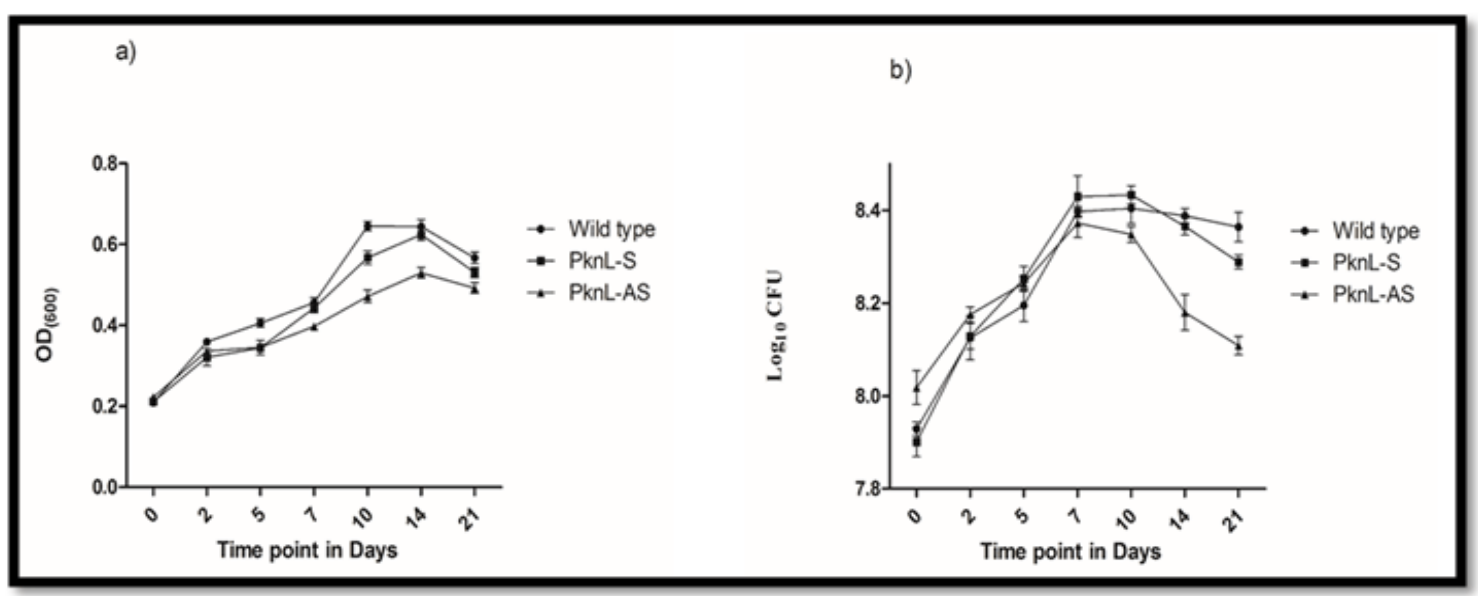

Figure 3: In vitro growth and survival kinetics in Carbon starved medium (CS)

Growth and viability of the strains Wild type, PknL-S and PknL-AS in CS media were monitored by measuring the $\mathrm{OD}_{600}$ and CFU obtained from serial dilutions from enriched 7H10 Agar plates with 10mM IPTG on 0,2,5,7,10,14 \& 21 days. The data is representative of three independent experiments. Error bars represent the standard error of the means. The graph represents the

a) $\mathrm{OD}_{600}$ plotted against the respective time points in CS media and

b) $\log 10(\mathrm{CFU} / \mathrm{ml})$ of the strains grown in enriched $7 \mathrm{H} 10$ agar plates.

There was no significant difference between the strains in growth but the viability of PknL-AS in nutrient rich 7H10 solid medium showed a significant decrease $(\mathrm{p}<0.01$ to $\mathrm{p}<0.001)$ compared to PknL-S and Wild type on day 14 \& 21 .

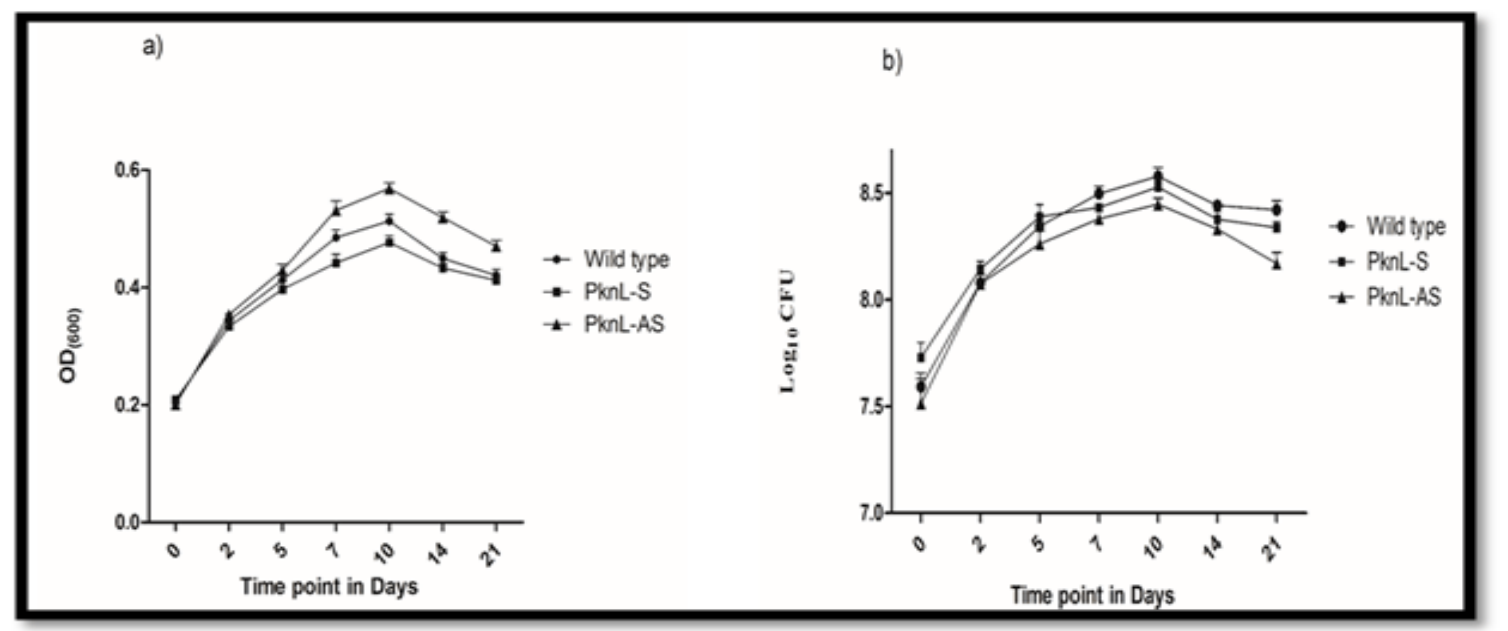

Figure 4: In vitro growth and survival kinetics in Nitrogen starved medium supplemented with ammonia (NSA)

Growth and viability of the strains Wild type, PknL-S and PknL-AS in NSA media were monitored by measuring the $\mathrm{OD}_{600}$ and CFU obtained from serial dilutions from enriched 7H10 Agar plates with $10 \mathrm{mM}$ IPTG on 0,2,5,7,10,14 \& 21 days. The data is representative of three independent experiments. Error bars represent the standard error of the means. The graph represents the

a) $\mathrm{OD}_{600}$ plotted against the respective time points in NSA media and

b) $\log 10(\mathrm{CFU} / \mathrm{ml})$ of the strains grown in enriched $7 \mathrm{H} 10$ agar plates.

There was no significant difference between the growth in starvation medium and viability of the strains in nutrient rich 7H10 solid medium.

type, PknL-S and PknL-AS were able to revive its growth following starvation. When closely observed, the survival pattern of Wild type and PknL-AS were same whereas PknL-S seemed slightly retarded in growth which was not significant (Figure 4b).

\section{In vitro growth kinetics and survival pattern in Nitrogen starved medium (NSG)}

In order to assess the growth and viability of the strains in the presence of glutamic acid as the sole nitrogen source, the growth profile of Wild type, PknL-S and PknL- AS was compared by monitoring the $\mathrm{OD}_{600}$ in modified 7H9 medium, containing only an organic source of nitrogen (NSG) and the viability of the strains were assessed by comparing the CFU obtained after transferring the serially diluted cultures of starved medium in nutrient rich 7H10 agar supplemented with OADC at 0,2,5,7,10,14 and 21 days. Here again, all the strains showed a similar growth profile in the presence of glutamic acid, but PknL-S showed a slightly enhanced (not significant) growth after day 5 compared to Wild type and PknL-AS (Figure 5a). This observation is quite controversial to the one seen in 7H9NSA. The viability of the strains PknL-S 


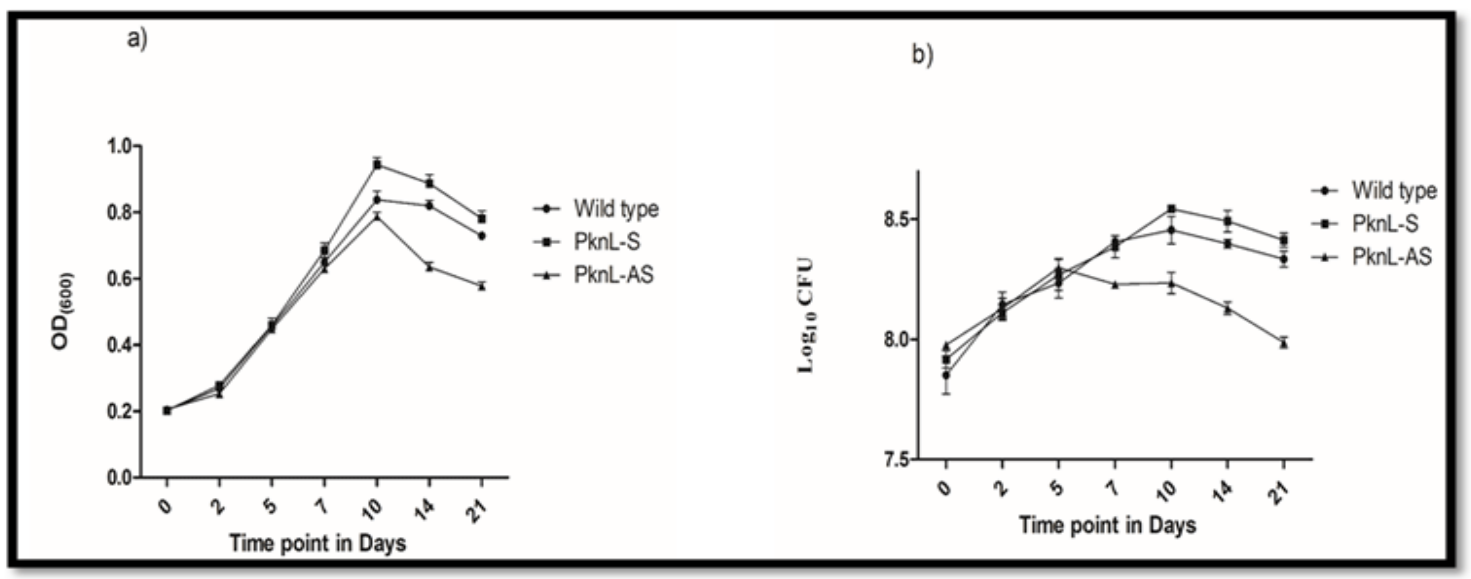

Figure 5: In vitro growth and survival kinetics in Nitrogen starved medium supplemented with glutamic acid (NSG)

Growth and viability of the strains Wild type, PknL-S and PknL-AS in NSG media were monitored by measuring the $\mathrm{OD}_{600}$ and CFU obtained from serial dilutions from enriched 7H10 Agar plates with $10 \mathrm{mM}$ IPTG on 0,2,5,7,10,14 \& 21 days. The data is representative of three independent experiments. Error bars represent the standard error of the means. The graph represents the

a) $\mathrm{OD}_{600}$ plotted against the respective time points in NSG media and

b) $\log 10(\mathrm{CFU} / \mathrm{ml})$ of the strains grown in enriched $7 \mathrm{H} 10$ agar plates.

There was no significant difference between the strains in growth but the viability of PknL-AS in nutrient rich 7H10 solid medium showed a significant decrease $(p<0.05$ to $p<0.001)$ compared to PknL-S and Wild type from day 10 onwards.

showed an increased survival capacity followed by Wild type and then PknL-AS. There was a significant $(p<0.01)$ difference observed between the viability of PknL-S and PknL-AS on day 14 \& 21 (Figure 5b).

\section{In vitro growth kinetics and survival pattern in $7 \mathrm{H} 9$ inhibited with glutamine inhibitor L-Methionine Sulfoximine (NSMSX)}

To decipher the growth and viability of the strains in the presence of a Glutamine inhibitor, L-Methionine Sulfoximine
(MetSox), the growth profile of Wild type, PknL-S and PknLAS was compared by monitoring the $\mathrm{OD}_{600}$ in $7 \mathrm{H} 9$ medium containing 200uM of MetSox (NSMSX) and the viability of the strains were assessed by comparing the CFU in nutrient rich 7H10 agar supplemented with OADC at 0, 2,5,7,10,14 and 21 days. A comparative reduction in the growth of PknL-S to Wild type and PknL-AS was observed. It was also interesting to note that PknL-AS though did not show any significant difference, was able to survive in the nitrogen starved medium induced by the addition of MetSox comparatively better than the other two

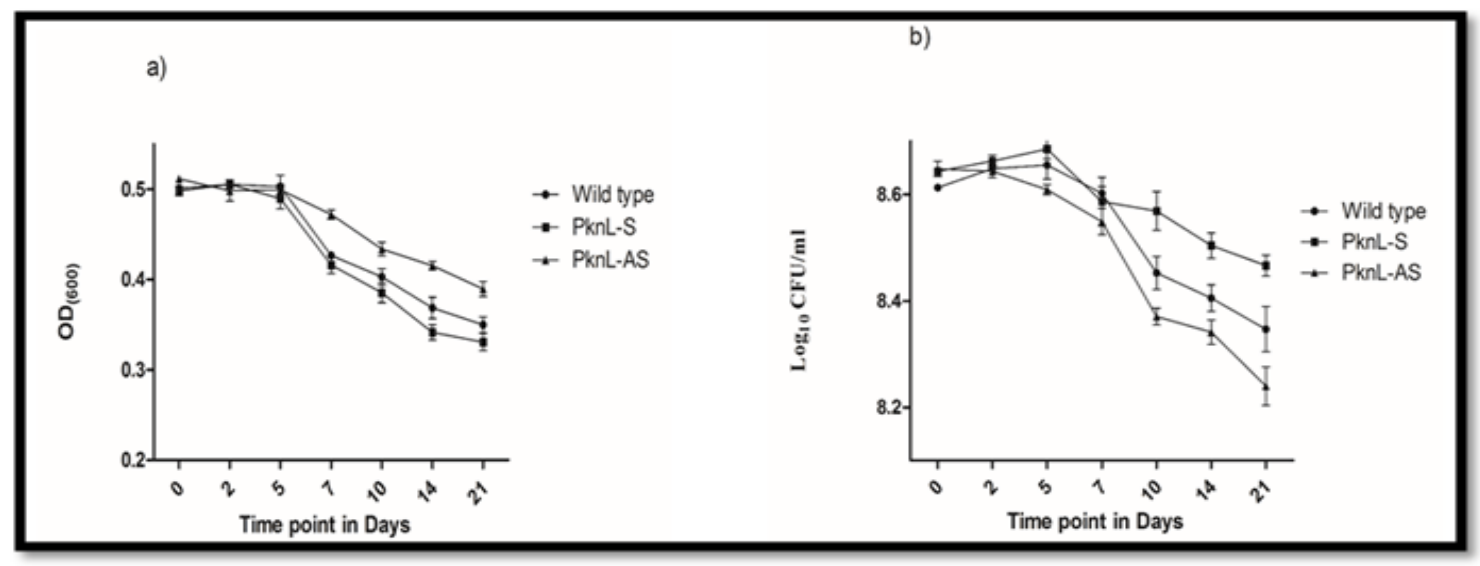

Figure 6: In vitro growth and survival kinetics in 7H9 medium with MSX (NSMSX)

Growth and viability of the strains Wild type, PknL-S and PknL-AS in NSMSX media were monitored by measuring the $\mathrm{OD}_{600}$ and CFU obtained from serial dilutions from enriched 7H10 Agar plates with 10mM IPTG on 0,2,5,7,10,14 \& 21 days. The data is representative of three independent experiments. Error bars represent the standard error of the means. The graph represents the

a) $\mathrm{OD}_{600}$ plotted against the respective time points in NSMSX media and

b) $\log 10(\mathrm{CFU} / \mathrm{ml})$ of the strains grown in enriched $7 \mathrm{H} 10$ agar plates.

There was no significant difference between the strains in growth but the viability of PknL-S in nutrient rich 7H10 solid medium showed a significant increase ( $\mathrm{p}<0.01$ to $\mathrm{p}<0.001)$ compared to PknL-AS and $(\mathrm{p}<0.05)$ compared to Wild type from day 10 onwards. 
strains (Figure 6a). However, the viability of strains depicted a different scenario. Although PknL-AS survived better in starved conditions, the viability of PknL-AS in nutrient rich 7H10 agar was not able to match the survival pattern of PknL-S which was much enhanced than the other two strains (Figure 6b). But, the CFU performed on 7H10 Agar with MetSox, completely inhibited the growth of PknL-S from day 7, followed by Wild type and then PknL-AS until day 14. Since there were no colonies observed in PknL-S, after day 7, and for Wild type on day 10 onwards, the graph was done by assuming the colony count as 1 for PknL-S and Wild type for the respective time points. There were no colonies observed for PknL-AS after day 14, the graph is shown only up to day 14 instead of day 21 (Figure 7).

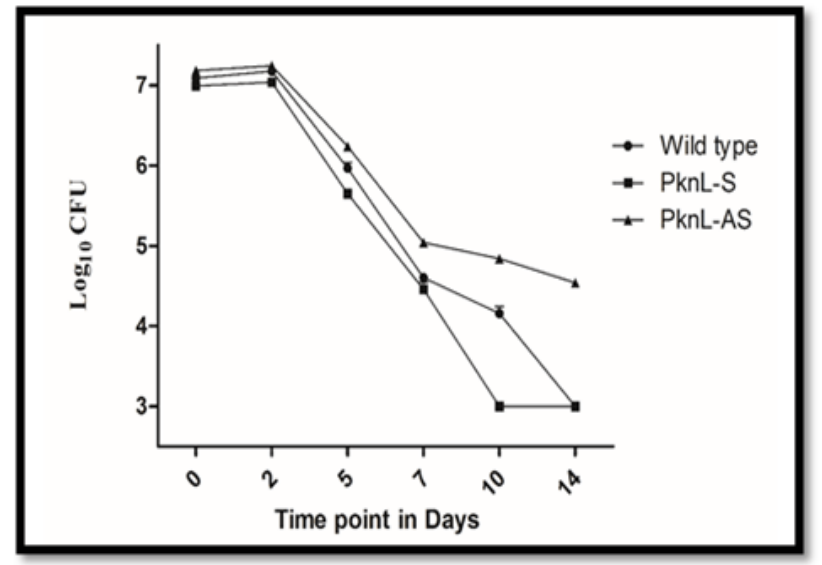

Figure 7: In vitro survival kinetics in 7H10 Agar with MSX (7H10MSX)

Viability of the strains Wild type, PknL-S and PknL-AS were enumerated with CFU obtained from serial dilutions from enriched 7H10 Agar plates with MSX and 10mM IPTG on 0,2,5,7,10 \&14 days. The data is representative of three independent experiments. Error bars represent the standard error of the means. The graph represents the $\log 10(\mathrm{CFU} / \mathrm{ml})$ of the strains grown in enriched 7H10MSX agar plates. There was a significant difference $(p<0.01$ to $p<0.001)$ observed between the viability of PknL-AS and the other two strains from day 10 onwards.

\section{Assessment of intracellular growth in infected THP-1 macrophages}

To determine whether the inhibition of $P k n L$ has any effect on the intracellular growth of the strains in THP-1 macrophages, the growth of all the strains PknL-AS, PknL-S along with the wild type strain were assessed by comparing the CFU in 7H10 Agar plates with 10\% OADC and supplemented with different concentrations of IPTG $(0-10 \mathrm{mM})$ on days $0,1,3,5 \& 7$. The intracellular growth of all the strains were similar in the absence of the inducer $(0 \mathrm{mM}$ IPTG), but the growth of PknL-AS decreased considerably in the presence of $1 \mathrm{mM}$ and $10 \mathrm{mM}$ IPTG concentration. The decrease in the viability of PknL-AS was much more significant $(p<0.001)$ on days 3 and 7 in the presence of $10 \mathrm{mM}$ IPTG compared to Wild type and PknL-S which signifies the fact that loss of $P k n L$ has led to reduction in the intracellular survival of the strain (Figure 8).

\section{Kinetics of IL-6 cytokine expression in differentiated THP-1 cells}

All the strains showed a significant increase in the expression of IL- 6 starting at 4 and $24 \mathrm{hrs}$ post infection than the uninfected control at all concentration of IPTG. Although a slight increase in the level of IL-6 expression was observed on days 3, 5 \& 7 in the absence of the inducer (0mM IPTG), it was still significantly less than the expression induced by Wild type, PknL-S and PknL-AS. We did not observe any significant difference in the expression level of IL- 6 induced by these strains. The expression levels of IL- 6 was at the highest on day 1 and considerably reduced in the following days irrespective of the nature of the infected strain (Figure 9).

\section{Kinetics of IL-12p40 cytokine expression in differentiated THP-1 cells}

The expression of IL-12p40 was observed in all the strains including the uninfected control irrespective of the presence or absence of the inducer. However, we observed a significant increase in the cytokine levels induced in THP-1 induced by PknLAS at $10 \mathrm{mM}$ IPTG concentration compared to PknL-S on day 3 and day 7. Similar to IL-6, the expression level of IL-12p40 was also at the highest on day 1 and showed a subsequent reduction in the following days (Figure 10).

\section{Discussion}

There exists a controversy regarding the location of $M$. tuberculosis in the absence of clinical disease leading to persistence and it is believed that the viable bacilli reside within fibrotic granulomatous lesions in the lungs which are maintained in a dormant state through conditions such as low oxygen tension and nutrient limitation. Hence many in vitro models and studies using conditions of reduced oxygen, nutrient deprivation and stationary phase survival have demonstrated that $M$. tuberculosis is able to survive for extended periods in a non-replicating state.

Serine/threonine kinases are generally involved in detecting and responding to the immediate changes in the environment through phosphorylation/dephosphorylation activity and we have already reported the role of $P k n L$ in the adaptive response of M. tuberculosis under the influence of environmental stress [11]. We extended our study in investigating the role of $P k n L$ in starvation survival response which may contribute to the 
a)

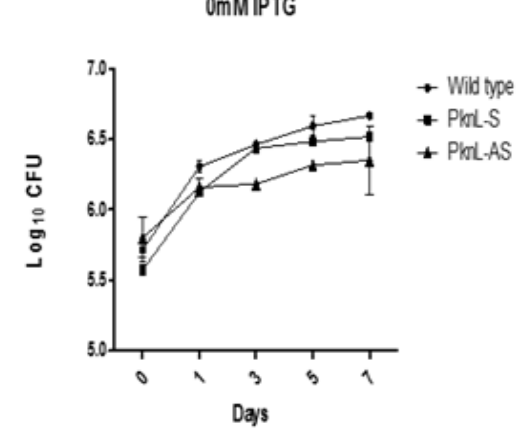

b)

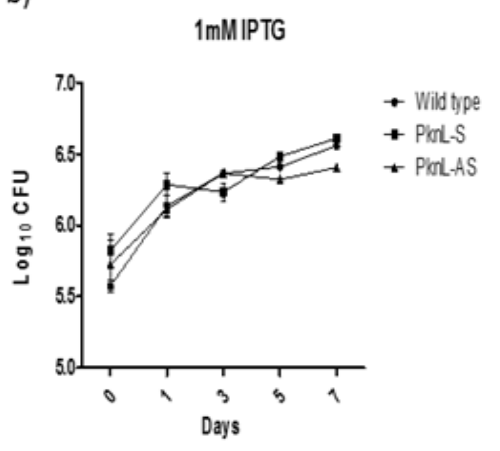

c)

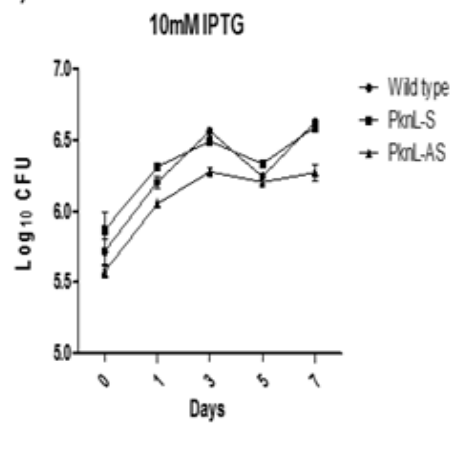

Figure 8: Intracellular growth rate in differentiated THP-1 macrophages

To visualize the viability of the intracellular bacteria PMA - differentiated THP-1 cells (1X106) were infected with Wild type, PknL-S and PknL-AS along with different concentrations of IPTG. Surviving intracellular bacteria were enumerated with CFU obtained from serial dilutions from cell lysates collected post infection at different time points. The graph represents the $\log 10(\mathrm{CFU} / \mathrm{ml})$ of the strains at

a) $0 \mathrm{mM}$ IPTG,

b) 1 mM IPTG and

c) $10 \mathrm{mM}$ IPTG on $0,1,3,5 \& 7$ days post infection.

The data is representative of three independent experiments. Error bars represent the standard error of the means. There was a significant difference $(p<0.01$ to $p<0.001)$ observed between the viability of PknL-AS and the other two strains on day 3 and 7 in the presence of $10 \mathrm{mM}$ IPTG.

a)

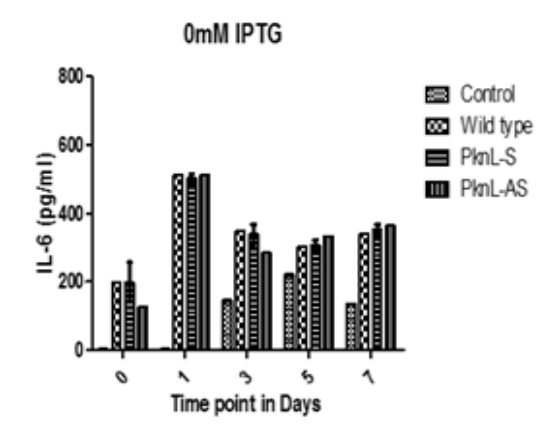

b)

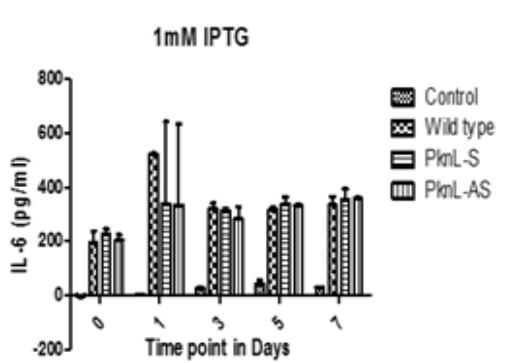

c)

$10 \mathrm{mM} \mathrm{IPTG}$

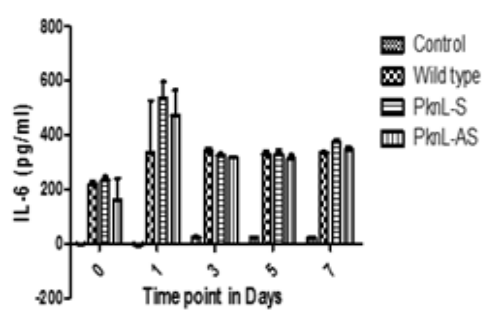

Figure 9: Kinetics of IL-6 production following infection

Level of IL- 6 expression was quantified by ELISA from the supernatants collected from THP-1 cells infected with Wild type, PknL-S and PknL-AS at different time points following infection. The graph represents the cytokine level (pg/ml) induced by these strains at

a) 0 mM IPTG,

b) $1 \mathrm{mM}$ IPTG and

c) $10 \mathrm{mM}$ IPTG on $0,1,3,5 \& 7$ days post infection.

The data is representative of three independent experiments. Error bars represent the standard error of the means. There was no significant difference in the cytokine level induced by these strains.

persistence of the mycobacteria inside the host. We have employed different starvation models which probably mimics the in vivo starvation conditions to elucidate the growth and survival kinetics of the PknL knockdown strain (PknL-AS), an over-expressed strain (PknL-S) and Wild type $\left(\mathrm{H}_{37} \mathrm{Rv}\right)$ which were previously generated. All the strains were initially grown in enriched 7H9 media supplemented with tween and ADS before transferring them to the respective starvation mediums and the growth was monitored by measuring the OD at $600 \mathrm{~nm}$. After starvation, the strains were again transferred to enriched 7H10 solid media and CFUs were enumerated to decipher their viability and reactivating capacity. All the strains Wild type, PknL-S and PknL-AS showed a reduction in their growth under Complete Nutrient Starvation (CNS media). But, the reactivation capacity of the Wild type and PknL-S was restored when transferred to nutrient rich Agar and a significant decrease in the viability of PknL-AS was observed. 
a)

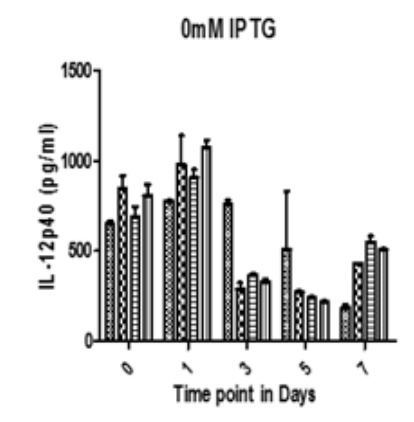

b)

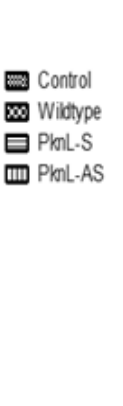

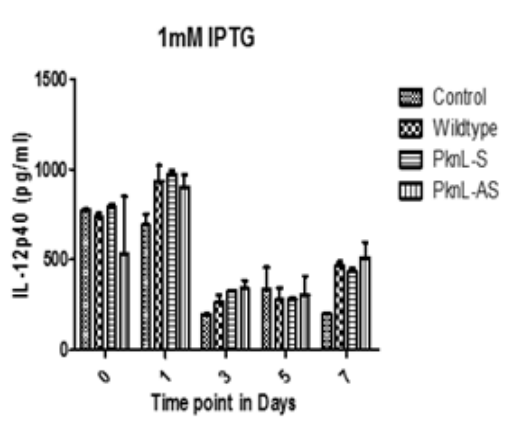

c)

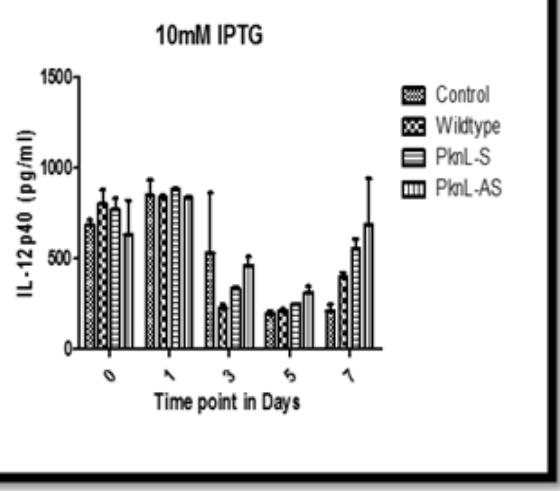

Figure 10: Kinetics of IL-12p 40 production following infection

Level of IL-12p40 expression was quantified by ELISA from the supernatants collected from THP-1 cells infected with Wild type, PknL-S and PknL-AS at different time points following infection. The graph represents the cytokine level $(\mathrm{pg} / \mathrm{ml})$ induced by these strains at

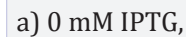

b) $1 \mathrm{mM} \mathrm{IPTG}$ and

c) $10 \mathrm{mM}$ IPTG on $0,1,3,5$ \& 7 days post infection.

The data is representative of three independent experiments. Error bars represent the standard error of the means. There was a significant increase in the level of cytokine induced by PknL-AS compared to PknL-S and Wild type on day 3 and 7 in the presence of 10mM IPTG.

Intraphagosomal M. tuberculosis may encounter inorganic phosphate $(\mathrm{Pi})$ depletion in addition to reactive oxygen and nitrogen intermediates and acid shock [14]. The Phosphate (pho) regulon, responsible for $\mathrm{Pi}$ management in bacteria is expressed during Pi starvation in E. coli and B. subtilis following a phospho- relay events between the sensor histidine kinase and response regulator pair of a 2-component regulatory system. This regulatory system is negatively regulated under high $\mathrm{Pi}$ conditions by the repressor PhoU [15]. Pi starvation is shown to induce the stringent response in E. coli, leading to RelA-mediated synthesis of the alarm one hyperphosphorylated guanosine, abbreviated (p) ppGpp [16]. The synthesis of (p) ppGpp in $M$. smegmatis and MTB is regulated by polyP via the mprA-SigE-relA pathway through transcriptional control of relA [17]. Pi starvation induced marked changes in bacillary morphology and acid-fast staining properties in MTB and the expression of SenX3-RegX3 was induced in Pi limiting conditions $[7,18]$. The growth of all the strains Wild type, PknL-S and PknL-AS in phosphate depleted (PS) media was almost similar until day 5 and plummeted down below $0.2 \mathrm{OD}_{600}$ on day 7. After which, PknL-S showed a steep increase in growth compared to PknL-AS and Wild type. Similarly, the viability of PknL-S showed a better response followed by Wild type in nutrient rich 7H10 Agar. Here again, we observed that PknL-AS was not able to match the reactivation pattern of Wild type and PknL-S.

Carbon plays a prominent role in the adaptation of $M$. tuberculosis. Many evidences exist to indicate that MTB strains which lack the components involved in the central carbon metabolism such as pyruvate dehydrogenase complex, glyoxylate shunt, or the gluconeogenic enzyme phosphoenolpyruvate carboxykinase were found to be attenuated and failed to replicate during the chronic phase of infection in a mouse model of pulmonary TB [19-22]. These studies reveal that M. tuberculosis has the capacity to derive carbon source from carbohydrates and lipids. However, the corresponding uptake protein for both the carbon sources inside the human host remains unknown for $M$. tuberculosis. The existing knowledge of carbon metabolism was derived from E. coli, which supports the fact that most bacteria consume each carbon source in a sequential order based on the rate at which it supports growth [23]. This phenomenon of diauxic growth is mediated by Carbon Catabolite Repression (CCR) [24]. Information based on the evidences pertaining to the growth of M. tuberculosis in different carbon sources has established a fact that MTB grows fastest in glycerol, followed by dextrose and acetate $[21,25,26]$. In this study, we assessed the growth of all the strains in the complete carbon depletion lacking all three key ingredients of carbon i.e. dextrose, glycerol and acetate. There was no significant difference between the growth profile of Wild type, PknL-S and PknL-AS in CS media. This observation might imply that $P k n L$ does not have a role in carbon starvation conditions. Tylaxopol was used instead of Tween 80 in the medium as it might compensate for the carbon deficiency as de-esterification of Tween 80 furnishes MTB with oleic acid serving as a carbon source [27]. These starved Mycobacterial strains were assessed for their viability by performing CFU in nutrient rich 7H10 Agar. PknL-S showed a better survival capacity compared to Wild type and PknL-AS and the viability of PknL-S was significantly higher compared to PknL-AS. Although, the experimental setup was not conducive enough to draw any conclusive role for $P k n L$ in carbon acquisition, the reactivation capacity of PknL-S was definitely better that Wild type and PknL-AS indicating the role of $P k n L$ in nutrient acquisition.

M. tuberculosis is a prototrophic bacterium which can assimilate inorganic compounds preferentially Ammonium as 
nitrogen sources for the synthesis of all of its nitrogen-containing constituents [28]. Ammonium is mainly transported into the cell by the Amt gene, located in an operon with GlnD and PII-encoding GlnK gene suggesting the involvement of all the three in the same process [29]. The function of this putative transporter, its virulence and physiology still remains to be determined although expression of Amt is found to be increased during nitrogen limitation [8].

Amino acids (organic source) also serve as the best organic source of nitrogen and have been recognized to support the in vitro growth of $M$. tuberculosis, especially glutamate and aspargine [30]. In order to be absorbed as a nitrogen source, amino acids undergo de-amination reactions leading to the release of ammonium [31,32]. Aspartate, glutamate, asparagine and glutamine are shown to be the most efficient in supporting M. tuberculosis growth in vitro and in acidic conditions when they are provided as sole nitrogen sources [33].

In order to assess the growth and viability of the strains, we initially grew the strains in normal 7H9 enriched media and then transferred to nitrogen starved media which consisted of all the composition of 7H9 media except for Ammonium Sulfate and L-glutamic acid and by replacing Ferric ammonium citrate with Ferric citrate. The initial $\mathrm{OD}_{600}$ was kept at 0.2 , as done for other starvation experiments but all the strains failed to grow following its transfer to nitrogen starved media and by day 21 , the $\mathrm{OD}_{600}$ was reduced to 0.04 which is very minimal (data not shown). All the strains were not able to revive and lost its viability even after it was transferred back to 7H10 nutrient rich Agar, due to which, the viability count following the nitrogen starvation became impossible. Hence, we performed another experiment by supplementing either Ammonium sulfate or L-glutamic acid as the sole nitrogen source and also to identify the preferential source of nitrogen acquisition of PknL. Here we observed that all the strains Wild type, PknL-S and PknL-AS were able to grow in either of the medium containing Ammonium sulfate or L-glutamic acid as the sole source of nitrogen.

This phenomenon indicates that $M$. tuberculosis has the capacity to exploit both ammonia (inorganic compound) and glutamine (organic compound) as nitrogen sources. By comparing the growth of Wild type, PknL-S and PknL-AS in presence of different nitrogen sources, it was observed that PknL-AS grew better in presence of ammonium sulfate and PknL-S grew better in the presence of L-glutamic acid. But a close observation shows that PknL-AS retained its growth between 0.5 to $0.8 \mathrm{OD}_{600}$ approximately in both the medium, whereas PknL-S grew well reaching up to approximately $1 \mathrm{OD}_{600}$ in the presence of L-glutamic acid. Although there was no significant difference in the growth between both the strains, the fact that PknL prefers glutamine over ammonia cannot be neglected. The same phenomenon was observed in $P k n L$ over expressed strain in $M$. smegmatis [34]. The presence of GlnD-Uridylyl transferase motif on the C-terminal of the gene could also contribute to the choice of nitrogen acquisition.
Another method of nitrogen starvation is inhibiting the activity of Glutamine Synthetase (GS) essential for nitrogen metabolism. L-methionine sulfoximine (MetSox) is a potent inhibitor of GS [35]. MetSox is phosphorylated by GS in the presence of ATP, which irreversibly binds to the active site preventing the entry of glutamate for nitrogen acquisition of bacteria. MetSox by itself is shown to prevent the growth of several Mycobacterial species and is capacitated to inhibit all the four GS present in M. tuberculosis [36, 37].

Since M. tuberculosis strains were not able to grow in media without any of the nitrogen sources, all the strains were allowed to grow in $7 \mathrm{H} 9$ enriched media until log phase to an $\mathrm{OD}_{600}$ of $\sim 0.5$ to which $200 \mu \mathrm{M}$ of MetSox was added. As expected the growth of the strains were observed to decrease following the addition of MetSox. But the strains were able to revive its growth when transferred to enriched 7H10 Agar. In order to confirm that the reduction in growth was mainly due to addition of MetSox, the viability count was performed on both 7H10 enriched agar plates with and without $200 \mu \mathrm{M}$ of MetSox. It was observed that PknL-S which showed a much decrease in growth in the presence of MetSox, but grew well in enriched media. At the same time PknLAS which showed a comparatively better survival in presence of MetSox showed a comparatively lesser viability pattern in enriched 7H10 media which is more intriguing. However, all the strains were unable to grow in 7H10 plates containing MetSox as there was no growth observed for PknL-S after day 7, but there were 1 to 2 colonies observed for PknL-AS till day 14. Wild type strain showed growth until day 10 . Hence the CFUs were enumerated only until and day 14.

Irrespective of the mode of starvation, we observed that the growth of PknL-AS decreased when compared to PknL-S and Wild type. Similarly, the viability of PknL-AS was much less comparatively even upon transferring the strains to enriched media. Taken together these observations clearly indicate the importance of nitrogen for the growth of M. tuberculosis and it has the capacity to obtain nitrogen from either of the source. These results, albeit inconclusive point towards the role of $P k n L$ in nutrient acquisition.

Macrophages are routinely used to study the virulence of mycobacterial strains as the ability to grow inside macrophages indicates its virulence trait. We infected THP-1 differentiated macrophages with all these strains to observe their ability to survive inside macrophages. We observed that the growth of the knockdown strain PknL-AS was considerably reduced when compared to Wild type and PknL-S in the presence of both $1 \mathrm{mM}$ and $10 \mathrm{mM}$ concentration of IPTG indicating its impaired replication and persistence within the macrophages. This further supports the fact that $P k n L$ is required for the persistence of the pathogen inside the host.

Cytokine milieu secreted by macrophages are very important in orchestrating the immune response against M. tuberculosis. Subsequent to the intracellular growth rate we also investigated the expression of cytokines stimulated by these three different strains in THP-1 cell line. 
M.tb generally exploits IL-6 induction to interfere with host signaling pathways activated by IFN $-\gamma$ for its survival. It exploits IL 6 induction as one of the mechanisms to inhibit IFN- $\gamma$ [38]. The kinetics of IL- 6 secretion by these strains showed a very high level of expression on $1^{\text {st }}$ day of infection followed by a significant reduction in subsequent days. Almost all the strains showed a similar trend in the secretion of IL-6 and there was no difference in the cytokine expression induced by these strains at any time point.

The significantly high level of IL-12p40 expression was observed in THP-1 cells infected with PknL-AS when compared to PknL-S and Wild type. The high level of IL-12 induction is generally associated with loss in infectivity or lack of virulence of the infecting strain according to an earlier report which substantiates the fact that THP-1 macrophages infected with M. tuberculosis and M. bovis showed a similar reduction in the percentage of IL-12-producing cells when compared with attenuated BCG strain [39]. The low levels of IL-12p40 induction are associated with differential mode of infection strategy by strains for the establishment in the host environment. This speculation is supported by Manca, et al who have shown that highly pathogenic strains induce significantly lower levels of IL-12 in human monocytes when compared to less pathogenic strains [40].

In conclusion, our results demonstrate that $P k n L$ is required for growth under nutrient limiting conditions and is also required for the reactivating capacity of $M$. tuberculosis in enriched conditions. Further PknL is also essential of the intracellular survival of the pathogen and assists the pathogen in establishing a successful infection by persisting within the host. Unraveling the key pathways that are involved in nutrient acquisition during infection might provide new clues for designing innovative therapies. Identifying proteins that are involved in the transport of these respective nutrients will also constitute novel drug targets.

\section{Acknowledgement}

Dr. Ahmed Kabir Refaya would like to acknowledge Indian Council for Medical Research (ICMR) for providing Senior Research Fellowship.

\section{References}

1. Getahun H, Chaisson RE, Raviglione M. Latent Mycobacterium tuberculosis Infection. N Engl J Med. 2015;373(12):1179-1180. doi: 10.1056/NEJMc1508223

2. Wayne LG, Hayes LG. An in vitro model for sequential study of shiftdown of Mycobacterium tuberculosis through two stages of nonreplicating persistence. Infect Immun. 1996;64(6):2062-2069.

3. Cunningham AF, Spreadbury CL. Spreadbury, Mycobacterial stationary phase induced by low oxygen tension: cell wall thickening and localization of the 16-kilodalton alpha-crystallin homolog. J Bacteriol. 1998;180(4):801-808.

4. Hutter B, Dick T. Up-regulation of narX, encoding a putative 'fused nitrate reductase' in anaerobic dormant Mycobacterium bovis BCG. FEMS Microbiol Lett. 1999;178(1):63-69.
5. Nyka W. Studies on the effect of starvation on mycobacteria. Infect Immun. 1974;9(5):843-850.

6. R. O. Loebel, E. Shorr, H. B. Richardson. The Influence of Adverse Conditions upon the Respiratory Metabolism and Growth of Human Tubercle Bacilli. J Bacteriol. 1933;26(2):167-200.

7. Rifat D, Bishai WR, Karakousis PC. Phosphate depletion: a novel trigger for Mycobacterium tuberculosis persistence. J Infect Dis. 2009;200(7):1126-1135. doi: 10.1086/605700

8. Read R, Pashley CA, Smith D, Parish T. The role of GlnD in ammonia assimilation in Mycobacterium tuberculosis. Tuberculosis (Edinb). 2007;87(4):384-390. doi: 10.1016/j.tube.2006.12.003

9. Primm TP, Andersen SJ, Mizrahi V, Avarbock D, Rubin H, Barry CE. The stringent response of Mycobacterium tuberculosis is required for long-term survival. J Bacteriol. 2000;182(17):4889-4898.

10.Zhang M, Gong J, Lin Y, Barnes PF. Growth of virulent and avirulent Mycobacterium tuberculosis strains in human macrophages. Infect Immun. 1998;66(2):794-799.

11. Refaya AK, Sharma D, Kumar V, Bisht D, Narayanan S. A Serine/ threonine kinase $P k n L$, is involved in the adaptive response of Mycobacterium tuberculosis. Microbiol Res. 2016;190:1-11.

12. Haydel SE, Malhotra V, Cornelison GL, Clark-Curtiss JE. The prrAB two-component system is essential for Mycobacterium tuberculosis viability and is induced under nitrogen-limiting conditions. J Bacteriol. 2012;194(2):354-361. doi: 10.1128/JB.06258-11

13.Amon J, Bräu T, Grimrath A, Hänssler E, Hasselt K, Höller M, et al., Nitrogen control in Mycobacterium smegmatis: nitrogendependent expression of ammonium transport and assimilation proteins depends on the OmpR-type regulator GlnR. J Bacteriol. 2008;190(21):7108-7116. doi:10.1128/JB.00855-08

14. Rengarajan J, Bloom BR, Rubin EJ. Genome-wide requirements for Mycobacterium tuberculosis adaptation and survival in macrophages. Proc Natl Acad Sci USA. 2005;102(23):8327-8332. doi: 10.1073/pnas.0503272102

15.Lamarche MG, Wanner BL, Crépin S, Harel J. The phosphate regulon and bacterial virulence: a regulatory network connecting phosphate homeostasis and pathogenesis. FEMS Microbiol Rev. 2008;32(3):461-73.

16.Spira B, Silberstein N, Yagil E. Guanosine 3',5'-bispyrophosphate (ppGpp) synthesis in cells of Escherichia coli starved for Pi. J Bacteriol. 1995;177(14):4053-4058.

17. Sureka K, Dey S, Datta P, Singh AK, Dasgupta A, Rodrigue S, et al. Polyphosphate kinase is involved in stress-induced mprAB-sigE-rel signalling in mycobacteria. Mol Microbiol. 2007;65(2):261-276. doi: 10.1111/j.1365-2958.2007.05814.x

18. Glover RT, Kriakov J, Garforth SJ, Baughn AD, Jacobs WR. The twocomponent regulatory system senX3-regX3 regulates phosphatedependent gene expression in Mycobacterium smegmatis. J Bacteriol. 2007;189(15):5495-5503. doi: 10.1128/JB.00190-07

19. Marrero J, Rhee KY, Schnappinger D, Pethe K, Ehrt S. Gluconeogenic carbon flow of tricarboxylic acid cycle intermediates is critical for Mycobacterium tuberculosis to establish and maintain infection. 
Proc Natl Acad Sci USA. 2010;107(21):9819-9824. doi: 10.1073/ pnas. 1000715107

20. McKinney JD, Höner zu Bentrup K, Muñoz-Elías EJ, Miczak A, Chen B, Chan WT, et al., Persistence of Mycobacterium tuberculosis in macrophages and mice requires the glyoxylate shunt enzyme isocitrate lyase. Nature. 2000;406(6797):735-738. doi: $10.1038 / 35021074$

21. Elias EJM, McKinney JD. Mycobacterium tuberculosis isocitrate lyases 1 and 2 are jointly required for in vivo grow th and virulence. Nat Med. 2005;11(6):638-644. doi: 10.1038/nm1252

22.Shi S, S. Ehrt. Dihydrolipoamide acyltransferase is critical for Mycobacterium tuberculosis pathogenesis. Infect Immun. 2006. 74(1):56-63. doi: 10.1128/IAI.74.1.56-63.2006

23. Kovárová-Kovar K, Egli T. Growth kinetics of suspended microbial cells: from single-substrate-controlled growth to mixed-substrate kinetics. Microbiol Mol Biol Rev. 1998;62(3):646-666.

24.Görke B, Stülke J. Carbon catabolite repression in bacteria: many ways to make the most out of nutrients. Nat Rev Microbiol. 2008;6(8):613-624. doi: 10.1038/nrmicro1932

25.Youmans AS, Youmans GP. Studies on the metabolism of Mycobacterium tuberculosis. IV. The effect of fatty acids on the grow th of M. tuberculosis var. hominis. J Bacteriol. 1954;67(6):731733.

26. Youmans GP, Youmans AS. Studies on the metabolism of Mycobacterium tuberculosis. I. The effect of carbohydrates and alcohols on the growth of Mycobacterium tuberculosis var. hominis. J Bacteriol. 1953;65(1):92-95.

27. Dubos RJ, Davis BD. Factors Affecting the Growth of Tubercle Bacilli in Liquid Media. J Exp Med.1946;83(5):409-423.

28. Niederweis M. Nutrient acquisition by mycobacteria. Microbiology. 2008;154(Pt 3):679-692. doi: 10.1099/mic.0.2007/012872-0

29. Cole ST, Brosch R, Parkhill J, Garnier T, Churcher C, Harris D, et al. Deciphering the biology of Mycobacterium tuberculosis from the complete genome sequence. Nature. 1998;393(6685):537-544. doi: $10.1038 / 31159$

30. Lofthouse EK, Wheeler PR, Beste DJ, Khatri BL, Wu H, Mendum TA, et al., Systems-based approaches to probing metabolic variation within the Mycobacterium tuberculosis complex. PLoS One. 2013;8(9):e75913. doi: 10.1371/journal.pone.0075913
31. Sürken M, Keller C, Röhker C, Ehlers S, Bange FC. Anaerobic arginine metabolism of Mycobacterium tuberculosis is mediated by arginine deiminase $(\operatorname{arcA})$, but is not essential for chronic persistence in an aerogenic mouse model of infection. Int J Med Microbiol. 2008;298(7-8):657-661. doi: 10.1016/j.ijmm.2007.09.003

32.Viljoen AJ, Kirsten CJ, Baker B, van Helden PD, Wiid IJ. The role of glutamine oxoglutarate aminotransferase and glutamate dehydrogenase in nitrogen metabolism in Mycobacterium bovis BCG. PLoS One. 2013;8(12):e84452. doi: 10.1371/journal. pone. 0084452

33.Song H, Niederweis M. Niederweis, Uptake of sulfate but not phosphate by Mycobacterium tuberculosis is slower than that for Mycobacterium smegmatis. J Bacteriol, 2012;194(5):956-964. doi: 10.1128/JB.06132-11

34.Harini Lakshminarayan, A Rajaram, Sujatha Narayanan. Involvement of Serine Threonine Protein Kinase, PknL, from Mycobacterium Tuberculosis H37Rv in Starvation Response of Mycobacteria. Journal of Microbial \& Biochemical Technology. 2009; Vol. 1 (1):030-036.

35.Ronzio RA. and A Meister. Phosphorylation of methionine sulfoximine by glutamine synthetase. Proc Natl Acad Sci USA, 1968;59(1):164-170.

36. Harth G, Horwitz MA. An inhibitor of exported Mycobacterium tuberculosis glutamine synthetase selectively blocks the growth of pathogenic mycobacteria in axenic culture and in human monocytes: extracellular proteins as potential novel drug targets. J Exp Med. 1999;189(9):1425-1436.

37. Harth G, Horwitz MA. Inhibition of Mycobacterium tuberculosis glutamine synthetase as a novel antibiotic strategy against tuberculosis: demonstration of efficacy in vivo. Infect Immun. 2003;71(1):456-464.

38. Martinez AN, Mehra S, Kaushal D. Kaushal, Role of interleukin 6 in innate immunity to Mycobacterium tuberculosis infection. J Infect Dis. 2013;207(8):1253-1261. doi: 10.1093/infdis/jit037

39. Mendoza-Coronel E, M Castanon-Arreola. Comparative evaluation of in vitro human macrophage models for mycobacterial infection study. Pathog Dis 2016;74(6). doi: 10.1093/femspd/ftw052

40.Manca C, Reed MB, Freeman S, Mathema B, Kreiswirth B, Barry $\mathrm{CE}$, et al. Differential monocyte activation underlies strainspecific Mycobacterium tuberculosis pathogenesis. Infect Immun. 2004;72(9):5511-5514. doi: 10.1128/IAI.72.9.5511-5514.2004 